\title{
RECENT DEVELOPMENTS IN THE YOUNG PLANETARY NEBULA HEN-1357
}

\author{
M. BOBROWSKY \\ CTA Inc., Rockville, $M D$
}

Observations over the past four decades have revealed significant changes in the spectrum of Hen-1357. Here we present HST images and spectra showing the most recent developments. In 1950, Henize saw only $\mathrm{H} \alpha$ in emission; but more recent observations by Parthasarathy et al. in 1992 showed strong forbidden lines consistent with a young planetary nebula. The spherically aberrated 1992 HST images, in which Bobrowsky first optically resolved the nebula, showed a compact nebula surrounding the central star. Nebular gas appeared most strongly concentrated in an ellipse with its major axis subtending 1."6 from NW to SE. If this ellipse is actually a circular ring viewed obliquely, then our line of sight is inclined from the symmetry axis by $5^{\circ}$. Above and below the ring of gas are two bubbles containing lower-density gas. At the tip of each bubble, there is a hole where the gas inside the bubbles has broken through and is now escaping. The windblown appearance is

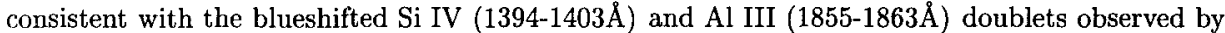

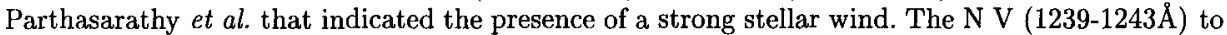
C IV (1548-1551 $\AA$ ) ratio has increased in recent years, consistent with a young nebula becoming increasingly ionized. Our recent (1996) spectra reveal additional developments that show the realtime development of this young nebula. Finally, the new HST Planetary Camera images of the nebula show detailed structure indicating a much more complex object than previously known, including the presence of a companion star 0.73 from the central star. 